\section{The therapeutic potential of modulating the ceramide/sphingomyelin pathway}

\author{
Richard Kolesnick
}

Laboratory of Signal Transduction, Sloan-Kettering Institute, Memorial Sloan-Kettering Cancer Center, 1275 York Avenue, New York, New York 10021, USA. Phone: (212) 639-7558; Fax: (212) 794-4342; E-mail: r-kolesnick@ski.mskcc.org.

J. Clin. Invest. 110:3-8 (2002). doi:10.1172/JCI200216127.

Membrane lipids of the sphingolipid class contain a long-chain sphingoid base backbone (such as sphingosine), an amide-linked, long-chain fatty acid, and one of various polar head groups. The structure of these head groups defines the various sphingolipid subtypes, with a hydroxyl group found in ceramide, phosphorylcholine in sphingomyelin (SM), and carbohydrates in glycosphingolipids (Figure 1 and not shown). Because SM is concentrated in the outer leaflet of the plasma membrane and provides a barrier to the extracellular environment (1), it was long assumed to serve only structural roles. However, in 1987 our laboratory reported for the first time rapid sphingomyelinase (SMase) activation in response to 1,2-diacylglycerols but not phorbol esters (2), and proposed the existence of an SM-based signaling pathway $(3,4)$. Subsequently, Hannun and coworkers showed that this pathway can be activated by receptor-mediated mechanisms (5) and provided evidence that ceramide is a second messenger (6). Indeed, sphingolipid metabolism has proved to be a dynamic process, and sphingolipid metabolites - including ceramide, sphingosine, and sphingosine 1-phosphate (S1P) - are now recognized as messengers playing essential roles in cell growth, survival, and death $(7,8)$.

\section{The generation of bioactive sphingolipids}

Figure 1 shows that ceramide can be formed through SMase-dependent catabolism of SM, as well as by de novo synthesis. SMases - specialized enzymes with phospholipase C activity - hydrolyze the phosphodiester bond of SM. Several isoforms of SMase can be distinguished by their different $\mathrm{pH}$ optima, and some but not all of these molecules have been identified at the molecular level. Neutral SMase (NSMase) and acid SMase (ASMase) are rapidly activated by diverse stress stimuli and promote an increase in cellular ceramide levels over a period of minutes to hours $(7,8)$. Alkaline SMase activity is found in the intestinal mucosa and bile and does not appear to participate in signal transduction.

ASMase was originally considered a strictly lysosomal enzyme because of its $\mathrm{pH}$ optimum at 4.5-5.0. However, an ASMase isoform was recently shown to localize to secretory vesicles near the plasma membrane (9) by a mechanism involving carbohydrate remodeling and to be secreted extracellularly (10-12). Liu and Anderson have observed the plasma membrane form of ASMase in caveolae, microdomains enriched in SM, and they have shown that IL- $1 \beta$ stimulates its activity in this compartment (12). Because $\mathrm{pH}$ and other factors regulate the on-off rate of substrate, rather than the catalytic activity of the enzyme (11), SM associated with LDL particles can be hydrolyzed by ASMase even at the neutral $\mathrm{pH}$ found at the cell surface and in caveolae.

NSMases ( $\mathrm{pH}$ optimum 7.4) are only now being defined at the molecular level (7), but it is clear from the residual activity seen in ASMase knockout mice that one or more distinct genes must encode such enzymes (13). NSMase activity is inhibitable by glutathione and is therefore derepressed under conditions of oxidative stress, when glutathione is depleted (14). Agents such as TNF- $\alpha$, which induce this state, lead to prolonged ceramide generation (14). FFAs may also enhance NSMase activity (15), and conditions that increase FFA levels, such as might occur in the gastrointestinal tract upon treatment with cyclooxygenase inhibitors, increase NSMase activity (16).

De novo ceramide biosynthesis requires coordinate action of serine palmitoyl transferase and ceramide synthase to generate ceramide. This process begins with the condensation of serine and palmitoyl-CoA to form 3-ketosphinganine $(7,8)$, which is reduced to the sphingoid base sphinganine and acylated by ceramide synthase to generate dihydroceramide. This compound is oxidized to ceramide by converting sphinganine to sphingosine by introduction of a trans-4,5 double bond. Alternately, this pathway may re-utilize the sphingosine released by sequential degradation of more complex sphingolipids for ceramide synthesis. This pathway can be stimulated by drugs and ionizing radiation and usually results in a prolonged ceramide elevation $(17,18)$.

Once generated, ceramide may amass or may be converted into a variety of metabolites (Figure 1). Phosphorylation by ceramide kinase $(7,8)$ generates ceramide 1-phosphate (not shown), while deacylation by either neutral or acid ceramidase - the products of different genes (19) - yields sphingosine, which may be phosphorylated by sphingosine kinase to S1P. Two distinct sphingosine kinases have been cloned. These two isoforms differ in temporal patterns of appearance during development, are expressed in different tissues, and possess distinct kinetic properties $(20,21)$, implying that they perform different cellular functions.

Ceramide may also be converted back to SM by transfer of phosphorylcholine from phosphatidylcholine to ceramide via SM synthase $(7,8)$. Alternatively, it can be glycosylated by glucosylceramide synthase to form glucosylceramide (not shown), which may be further mod- 
ified by various enzymes in the Golgi apparatus to form complex glycosphingolipids.

\section{Ceramide and S1P as second messengers regulating apoptosis}

While ceramide has been proposed as a messenger for events as diverse as differentiation, senescence, proliferation, and cell cycle arrest, a larger body of research has focused on its role in apoptosis $(7,8)$. Evidence supporting ceramide as a message for apoptosis induction is based on data from many cell systems and comes from several kinds of studies. First, agonist- and stress-induced increases in ceramide levels precede biochemical and morphologic manifestations of apoptosis in many systems. Second, increasing cellular ceramide by addition of natural ceramide, exogenous SMase, or pharmacologic agents that interfere with enzymes of ceramide metabolism mimics effects of stress on apoptosis induction. Third, genetic models, including Niemann-Pick Disease cells, Asmase ${ }^{-/-}$mice, and Glucosylceramide synthase ${ }^{-/-}$mice, manifest the predicted abnormalities in stress responses from the biochemical and cell biologic studies. In view of the fact that other sphingolipid metabolites (except perhaps sphingoid bases and GD3 in selected cells) are not cytotoxic $(7,8)$, it thus appears that ceramide per se is necessary and likely sufficient for some forms of cell stress-associated apoptosis. Interestingly, ceramide may serve as a stress response signal even in Saccharomyces cerevisiae, as yeast mutants incapable of rapid de novo synthesis of this sphingolipid class fail to adapt and to regrow at elevated temperatures, and instead undergo growth arrest. This defect is bypassed by exogenous sphingolipid, indicating an obligate requirement for sphingolipids for this response. Thus, ceramide/sphingolipid signaling may constitute a programmed stress response that predates apoptosis evolutionarily.

One proposal that may help explain how ceramide serves these diverse functions is that of the "S1P Rheostat" (22). Spiegel and coworkers based this proposal on the observation that ceramide and its metabolite S1P often have opposing effects on biologic outcome. While ceramide is often antiproliferative and proapoptotic, S1P has been implicated as a second messenger in cellular proliferation and survival (23), and in protection against ceramide-mediated apoptosis (22). Thus, it has been suggested that the balance between these two sphingolipid messages may be an important factor determining survival or death of mammalian cells (23). As sphingosine levels in mammalian cells are often one to two orders of magnitude lower than those of ceramide, the generation of substantial amounts of S1P requires additional sphingosine production. Typically, S1P is produced by the coordinate activation of SMase to generate ceramide, ceramidase to free up sphingosine, and sphingosine kinase (24). In fact, activation of ceramidase may be so robust in some cells that the ceramide levels never rise substantially, indicating that SM has been converted stoichiometrically to sphingosine. For this reason, activation of the SM pathway can never be ruled out by the failure to detect ceramide increases, unless measurements of SM, sphingosine, and S1P levels are also available.

The recent discovery of a set of cell surface S1P receptors, the EDG proteins, has further complicated the interpretation of S1P dynamics (24). It is not yet clear to what extent S1P functions by ligating these receptors, rather than by acting as an intracellular signal. The available data suggest that, by either mode, it antagonizes ceramide-mediated biologic responses. Other metabolic pathways may also contribute to the diverse action of ceramide in different cellular systems. In general, the ceramide backbone of sphingolipids is generated in the endoplasmic reticulum, and the head groups are added in the Golgi apparatus, after which the newly synthesized lipids are shuttled to various organelles. Sphingolipid-degrading enzymes, conversely, have usually been thought to localize to the endosomal-lysosomal system, but recent studies have detected isoforms of both the synthetic and the degradative enzymes in alternative compartments, where they appear to play distinctive roles. A mitochondrial form of ceramide synthase, for instance, is exclusively activated by ionizing radiation treatment of HeLa cells and appears requisite for efficient mitochondrial commitment to the apoptotic process (R. Kolesnick, unpublished results). Like-

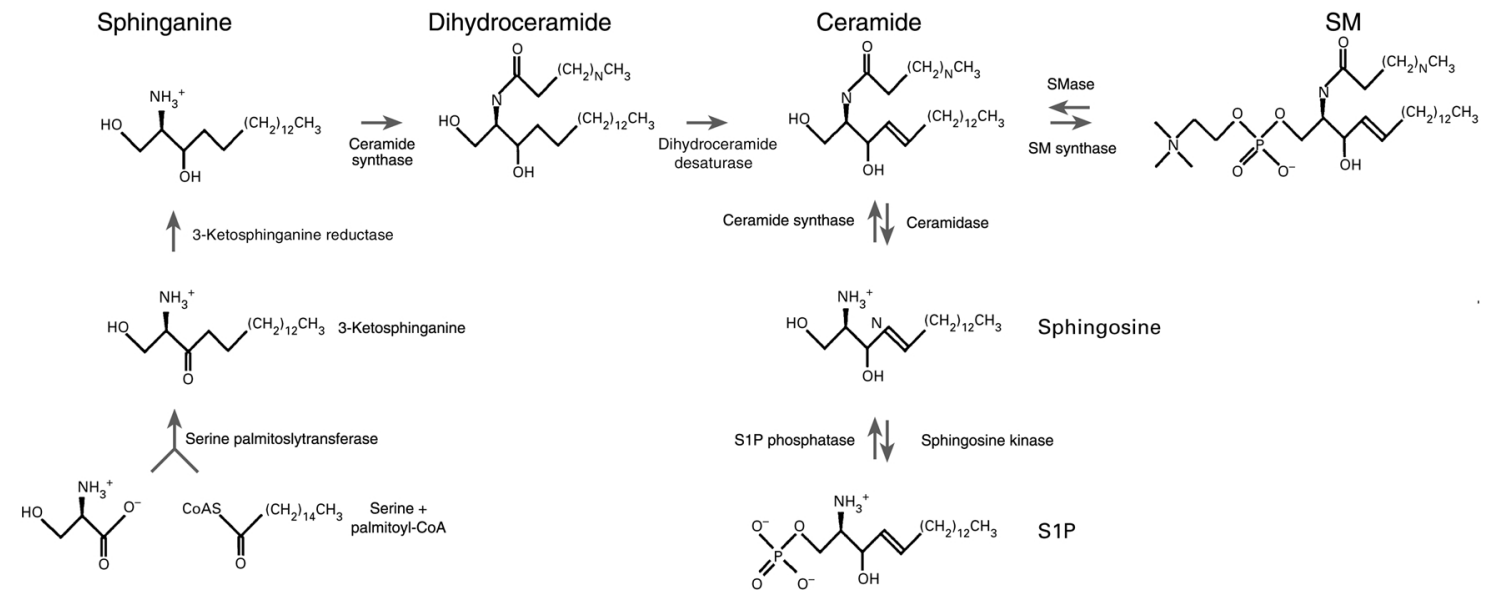

Figure 1

Schematic representation of sphingolipid intermediary metabolism. 


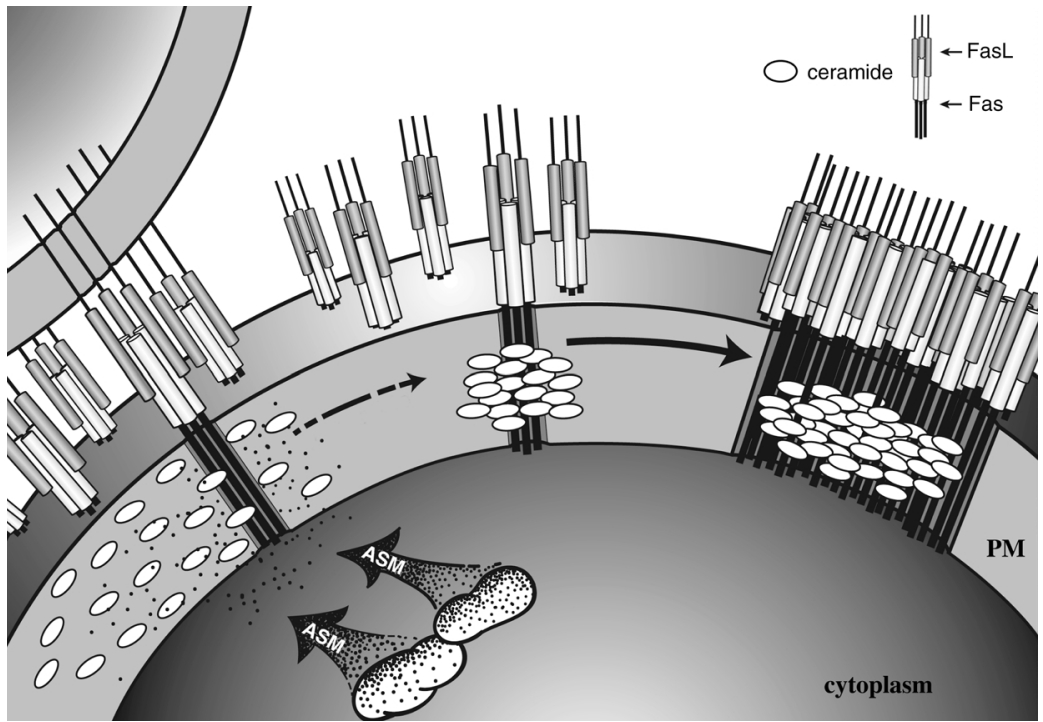

Figure 2

A model for Fas-induced capping through ASMase activation. Ligation of preformed Fas trimers on a target cell by transmembrane Fas ligand (FasL) activates small amounts of caspase- 8 within the cytoplasm of that cell, which is nonetheless sufficient to induce ASMase translocation into membrane rafts. The consequent hydrolysis of SM to ceramide, which selfassociates, initiates coalescence of ceramide-enriched rafts into larger patches (isolated ovals) and platforms (aggregated ovals). Only ligated Fas can enter and concentrate within these platforms, permitting oligomerization of the downstream Fas effectors FADD and caspase8 (not shown), the constituents of the death-inducing signaling complex (DISC). The extent to which this raft reorganization system is used for Fas signaling is currently unknown. Fas may not be the only cell surface receptor to employ this concentrating system, as a recent study suggests that CD40 may also cap in ceramide-rich platforms (61). Note that the transmembrane domains of some, and the cytoplasmic domain of all, Fas molecules are not depicted in this schematic for reasons of clarity. Similarly, the FasL-presenting cell is only shown once (upper left). PM, plasma membrane.

wise, both ASMase and SM synthase are targeted in part to the plasma membrane, with the former specifically targeted to membrane rafts (see below). One form of acid ceramidase may also be a secreted enzyme, while a form of neutral ceramidase may be mitochondrial (25) and hence might affect ceramide synthase-mediated ceramide signaling in that compartment.

\section{The biophysical basis of ceramide signaling}

In the last few years, a number of groups have generated ceramide in model membrane systems and described properties that fundamentally alter our understanding of how this lipid might act as a second messenger. Two properties, the ability to spontaneously form microdomains and the ability to act as a fusagen, seem particularly relevant to ceramide action (for review see ref. 26).

The interaction of sphingolipids, especially SM and cholesterol, drives the formation of plasma membrane rafts (also known as glycosphingolipid-enriched microdomains [GEMs] or detergent-insoluble glycosphingolipid-enriched microdomains [DIGs]). These rafts, formed in the Golgi apparatus, are targeted to the plasma membrane, where they are thought to exist as floating islands within the sea of bulk membrane. Although there is substantial disagreement as to their content, these rafts are considered in most reports to comprise
3500 lipid molecules and about 30 proteins (27). As much as $70 \%$ of all cellular $\mathrm{SM}$ may be found in rafts (28). A general consensus has developed over the last few years that plasma membrane rafts represent signaling microdomains. Rafts containing the caveolin proteins manifest a unique flask-shaped structure by electron microscopy and may possess different properties than do rafts lacking caveolin.

Numerous studies now show that SM hydrolysis to ceramide, usually via ASMase, occurs within rafts (12, 29-31). Recent work with a new anticeramide IgM supports this contention (R. Kolesnick and E. Gulbins, unpublished results). Ceramide generated within rafts appears to alter raft structure in a manner consistent with the known physical properties of ceramide. Thus, patches of rafts enriched in ceramide become visible within seconds after translocation of ASMase onto the outer leaflet of the plasma membrane upon Fas activation of Jurkat cells and primary cultures of hepatocytes. These microdomains rapidly fuse into larger domains, termed "platforms," and form caps on the cell surface (Figure 2). The functional significance of this reorganization of membrane structure would appear to allow for protein oligomerization. Indeed, preformed Fas trimers (32) localize to these domains and appear to form higherorder structures, allowing for the oligomerization of the downstream adaptors FADD/MORT-1 and caspase-8. (While Peter and coworkers arrived at a different conclusion [ref. 33], subsequent studies appear to corroborate the original observation [refs. 34, 35]). This key step, which, at least in some systems, is necessary for transmembrane transmission of the Fas apoptotic signal, is suppressed under basal conditions because only the ligated Fas that activates ASMase appears capable of entering the membrane platforms.

Other receptors, such as E-selectin and $\beta$-integrin, that cap in T cells but do not activate this raft reorganization system signal via ceramide-independent mechanisms. Whether environmental stresses similarly use this ceramide-dependent raft system for transmembrane signaling is currently unknown. Further, it is not certain whether modes of ceramide generation other than via ASMase can signal raft reorganization, or whether ceramide generated in other membranes serves oligomerizing function. Clearly additional work is necessary to explore this remarkable mode of cellular activation.

\section{Cellular targets for ceramide}

Ceramide directly regulates ceramide-activated protein phosphatases (CAPPs) (36), a ceramide-activated protein 

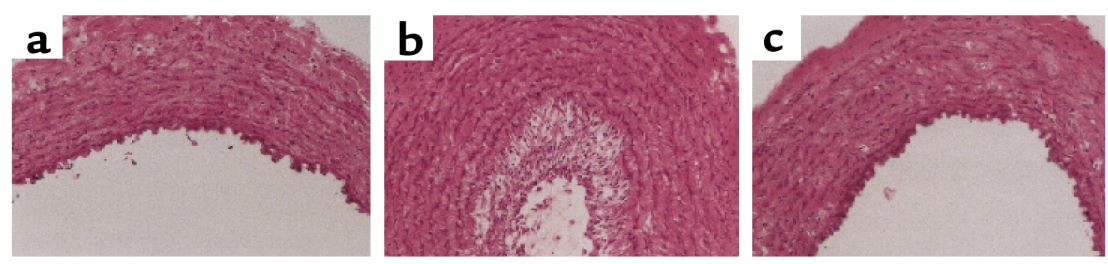

\section{Figure 3}

$\mathrm{C}_{6}$-ceramide limited neointimal hyperplasia after balloon angioplasty in rabbit carotid arteries. (a-c) Hematoxylin and eosin-stained sections from excised arteries 2 weeks after angioplasty. (a) Sham-treated control artery. (b) Artery treated with a balloon coated with DMSO/ethanol (1:1, vol/vol) displays marked neointimal hyperplasia. (c) Artery treated with a $\mathrm{C}_{6}$-ceramide-coated balloon displays minimal neointimal hyperplasia. Bar, 200 $\mu \mathrm{m}$. Reprinted with permission (49).

kinase (identical to kinase suppressor of Ras [KSR]) (37, 38 ), phospholipase $\mathrm{A}_{2}$ (39), cathepsin $\mathrm{D}(40)$, various protein kinase C (PKC) isoforms (41), and c-Raf-1 $(42,43)$. Both PP1 and PP2a are CAPPS that are conserved through yeast, which, in response to ceramide elevation, induce growth arrest or apoptosis, depending on the cellular context. Although the exact substrates are uncertain, evidence points to PKB/AKT1 (44), Bcl-2 (45), and SR proteins (36) as targets. KSR is a Ser/Thr kinase that phosphorylates c-Raf- 1 to initiate signaling through the mitogen-activated protein kinase cascade in response to ceramide elevation, TNF- $\alpha$, or epidermal growth factor, depending on cell type (for alternate view see ref. 46). ASMase-released ceramide binds directly to $\mathrm{PLA}_{2}$ and cathepsin D $(39,40)$. Binding of ceramide to cathepsin D in endosomes or in vitro triggers autocatalytic cleavage of cathepsin to its active form. A link between cathepsin D and apoptosis was originally defined by Kimchi and coworkers (47), who showed that cells transfected with antisense cathepsin D mRNA survive exposure to apoptotic stimuli. Thus, cathepsin D links ceramide to at least one form of apoptosis induction. The physiologic role of ceramide in $\mathrm{PLA}_{2}$ activation remains uncertain.

A recent study by Saito et al. may explain how ceramide regulates the activity of some of its substrates (48). By mutagenesis and deletion analysis of PKC $\delta$ and $\varepsilon$, these investigators showed that the conserved region (C1) - and particularly the cysteine loop region $\mathrm{C} 1 \mathrm{~B}-$ is the site of ceramide activation. Interestingly, numerous other proteins, including such known ceramideresponsive factors as KSR and c-Raf-1, contain C1B domains. Whether the $\mathrm{C} 1 \mathrm{~B}$ domain targets ceramideactivated proteins to sites of ceramide generation, such as plasma membrane rafts, is presently unknown.

\section{The potential for ceramide-based therapies}

Over the past few years there has been an escalating interest in exploring the role of ceramide and its metabolites in tissue physiology and pathophysiology. Levels of ceramide and S1P can be manipulated by various drugs, which are now being examined in clinical and preclinical studies. Knowledge of the cell type-specific activation of sphingolipid-dependent signaling pathways, derived from a large body of biochemical, genetic, and physiological studies, provides a strong impetus for this work. Typically, strategies that elevate cellular ceramide are being used for therapies aimed to arrest growth or promote apoptosis. Conversely, agents that reduce ceramide or elevate S1P tend to attenuate apoptosis and promote proliferation. One dramatic demonstration of the first approach is seen in the work of Kester and coworkers, who found that ceramide analogs, applied directly to damaged arteries, can be strongly antiproliferative (49). Neointimal hyperplasia of vascular smooth muscle cells and secondary occlusion of coronary arteries, the cause of restenosis after balloon angioplasty or stenting, affects nearly $20 \%$ of the 1.5 million patients who undergo coronary angioplasty yearly. Proliferation of cultured vascular smooth muscle cells appears to involve the extracellular signal-regulated kinase (ERK) and AKT kinase cascades and to be inhibited by ceramide (50). In vivo, $\mathrm{C}_{6}$-ceramide-coated balloon catheters prevent stretch-induced neointimal hyperplasia in rabbit carotid arteries (49) by inactivating ERK and AKT signaling and thereby inducing cell cycle arrest in stretch-injured vascular smooth muscle cells (50) (Figure 3).

Manipulation of ceramide levels may also enhance the effectiveness of some cancer therapies. Wanebo and coworkers showed that the addition of ceramide enhances taxol-mediated apoptotic death of Tu138 head and neck tumor cells (51). Preliminary data using Tu138 xenografts in nude mice showed synergistic reduction in tumor growth upon combined treatment (personal communication, $\mathrm{H}$. Wanebo). Clavien and colleagues have shown that the cellular content of ceramide in human colon cancer is reduced by more than $50 \%$ relative to that of healthy colon mucosa (52). The potent ceramidase inhibitor B13 increases the ceramide content of tumor cells and induces tumor cell apoptosis, without affecting the ceramide level or survival of normal liver cells. B13 also prevents growth of two aggressive human colon cancer cell lines metastatic to the liver. Together, these findings suggest that ceramidase inhibition offers a promising therapeutic strategy for selective toxicity toward malignant but not toward normal cells.

Blocking ceramide generation may also protect some tissues from side effects of cancer therapy. The lethal gastrointestinal syndrome, which limits the efficacy of radiation and chemotherapy (53), apparently results from apoptotic damage to the endothelial cells of the microvasculature of the small intestine, and, indirectly, from crypt stem cell dysfunction and tissue necrosis (54). Genetic inactivation of Asmase prevents this toxicity, as does treatment with the endothelial cell survival factor bFGF, which acts in part by suppressing ASMase activity. These studies suggest a potential approach to 
protecting the gut and increasing the therapeutic ratio.

Evidence from in vivo models demonstrates that pharmacologic manipulation of S1P may also be accomplished with therapeutic benefit. Tilly and coworkers showed that female Asmase - $^{-/}$mice are defective in the normal apoptotic deletion of fetal oocytes, leading to neonatal ovarian hyperplasia (55). Ex vivo, Asmase-oocytes and S1P-treated wild-type oocytes resist developmental and anticancer drug-induced apoptosis, confirming cell autonomy of the death defect. Moreover, in adult wild-type female mice, radiation-induced oocyte loss, the event that drives premature ovarian failure and infertility in female cancer patients, is completely prevented by injection of S1P into the ovary prior to irradiation. In ongoing mating trials, the pregnancy rates are $15 \%$ and $75 \%$ in female mice irradiated without and with prior in vivo ovarian S1P pretreatment, respectively (R. Kolesnick and J. Tilly, unpublished results). Critically, this preservation of fertility is accomplished without propagating genetic damage in offspring of the S1P-protected irradiated females. Thus, S1P may represent the first agent capable of protecting against the premature ovarian failure and infertility that are routine side effects observed in young girls and reproductive-age women treated for cancer.

Blockade of S1P biosynthesis provides another possible means to amplify ceramide-mediated death signals. Fenretinide (4-HPR), a synthetic retinoid that is cytotoxic for a variety of tumor cell lines in vitro, has recently been reported to induce sustained ceramide increases via the de novo pathway in cell lines of neuroblastoma and other cancer types, including leukemias $(56,57)$. Combining fenretinide with putative modulators of ceramide metabolism - such as glucosylceramide synthase inhibitors, which prevent the siphoning off of ceramide for glycosphingolipid synthesis, or safingol (L-threo-dihydrosphingosine), a sphingosine kinase inhibitor that prevents formation of S1P from degraded ceramide synergistically increases cytotoxicity in cell lines from various tumors, including those with altered p53 status and high alkylator resistance. Significantly, ceramide is only minimally increased in normal fibroblasts or blood mononuclear cells under similar conditions, and the toxicity to normal fibroblasts and myeloid progenitors from bone marrow appears to be slight (56). Clinically, it is likely that obtaining levels of fenretinide in tumors sufficient to achieve optimal ceramide generation will require intravenous delivery. Such formulations of fenretinide and safingol are currently being developed with support from the National Cancer Institute RAID program, and clinical trials are planned (56).

Previously published data likewise suggest that safingol may be useful in the clinic. Schwartz and coworkers administered this drug to patients with advanced cancer in a phase I clinical trial (58), either alone or in combination with doxorubicin, which is known to elevate ceramide in numerous transformed cell types. With a fixed dose of doxorubicin $\left(45 \mathrm{mg} / \mathrm{m}^{2}\right)$, safingol could be escalated to micromolar doses without dose-limiting toxicity and without altering the pharmacokinetics of doxorubicin. Encouraging clinical activity was observed in patients with pancreatic cancer and sarcoma.
Finally, Mandala et al. (59) have found that FTY720, a potent immunosuppressant that protects solid organ allografts in a number of experimental models through altered lymphocyte trafficking, is activated by phosphorylation to become a structural analog of S1P. Both S1P and phosphorylated FTY720 display similar affinity for and activation of S1P receptors. FTY720 has already passed through preclinical testing into clinical trials in renal transplant patients, in whom early data suggest that the drug is well tolerated and has a favorable toxicity profile (60). Thus lysophosphosphingolipids and their analogs may represent a promising new class of therapeutics for transplantation and autoimmune disorders.

\section{Conclusions}

An intrinsic function of the SM pathway is to respond to stress signals, whether environmental or pharmacologic, converting them into biochemical messages. Thus, by its very nature, it would appear that this mechanism is poised for therapeutic manipulation. Of particular interest is the observation in several systems that transformed cell types are hypersensitive to the effects of ceramide perturbation, suggesting that strategies to kill tumor cells by increasing their ceramide content or blocking S1P accumulation should have a favorable therapeutic index. The generality of this finding must still be tested in studies of many more normal and transformed cell types. Whether the differences observed in drug responses relate to alterations in tumoral sphingolipid handling, cell cycle regulation, or signal transduction remains an open question.

As evidenced by the recent paper of Mandala et al. in Science (59), the era of sphingolipid therapy seems near at hand (59). Successful modulation of this pathway in vivo depends on understanding cell type-specific responses to these messages in live tissue. For instance, the discovery that deletion of Asmase abrogates the normal developmental program of apoptosis in the oocyte has led to the promising discovery that $\mathrm{S} 1 \mathrm{P}$ might prevent sterility after exposure to cancer therapies. Now that the sphingolipid analog FTY720 has been identified as a legitimate pharmacologic agent for human patients, we can anticipate additional therapeutic success from the judicious use of sphingolipid derivatives, building on continuing preclinical studies with these agents.

\section{Acknowledgments}

The author would like to thank Gary Schwartz, C. Patrick Reynolds, Barry Maurer, Mark Kester, Harold Wanebo, James Ferrara, and Zvi Fuks for their consultation during preparation of this manuscript. The author is supported by NIH grants RO1 CA-42385 and CA-85704.

\footnotetext{
1. Kolesnick, R.N. 1991. Sphingomyelin and derivatives as cellular signals. Prog. Lipid Res. 30:1-38.

2. Kolesnick, R.N. 1987. 1,2-Diacylglycerols but not phorbol esters stimulate sphingomyelin hydrolysis in $\mathrm{GH}_{3}$ pituitary cells. J. Biol. Chem. 262:16759-16762.

3. Kolesnick, R.N., and Clegg, S. 1988. 1,2-Diacylglycerols, but not phorbol esters, activate a potential inhibitory pathway for protein kinase $\mathrm{C}$ in $\mathrm{GH}_{3}$ pituitary cells: evidence for involvement of a sphingomyelinase. J. Biol. Chem. 263:6534-6537.

4. Kolesnick, R.N. 1989. Sphingomyelinase action inhibits phorbol esterinduced differentiation of human promyelocytic leukemic (HL-60) cells. J. Biol. Chem. 264:7617-7623.
} 
5. Okazaki, T., Bell, R.M., and Hannun, Y. 1989. Sphingomyelin turnover induced by vitamin $\mathrm{D}_{3}$ in HL-60 cells: role in cell differentiation. J. Biol. Chem. 264:19076-19080

6. Okazaki, T., Bielawska, A., Bell, R.M., and Hannun, Y.A. 1990. Role of ceramide as a lipid mediator of $1 \alpha, 25$-dihydroxyvitamin $\mathrm{D}_{3}$-induced HL60 cell differentiation. J. Biol. Chem. 265:15823-15831.

7. Hannun, Y.A., Luberto, C., and Argraves, K.M. 2001. Enzymes of sphingolipid metabolism: from modular to integrative signaling. Biochemistry. 40:4893-4903

8. Mathias, S., Pena, L.A., and Kolesnick, R.N. 1998. Signal transduction of stress via ceramide. Biochem. J. 335:465-480.

9. Grassme, H., Schwarz, H., and Gulbins, E. 2001. Molecular mechanisms of ceramide-mediated CD95 clustering. Biochem. Biophys. Res. Commun. 284:1016-1030.

10. Schissel, S., Keesler, G., Schuchman, E., Williams, K., and Tabas, I. 1998. The cellular trafficking and zinc dependence of secretory and lysosomal sphingomyelinase, two products of the acid sphingomyelinase gene. $J$. Biol. Chem. 273:18250-18259.

11. Schissel, S., et al. 1998. Secretory sphingomyelinase, a product of the acid sphingomyelinase gene, can hydrolyze atherogenic lipoproteins at neutral $\mathrm{pH}$. Implications for atherosclerotic lesion development. J. Biol. Chem. 273:2738-2746.

12. Liu, P., and Anderson, R. 1995. Compartmentalized production of ceramide at the cell surface. J. Biol. Chem. 270:27179-27185.

13. Horinouchi, K., et al. 1995. Acid sphingomyelinase deficient mice: a model of types A and B Niemann-Pick disease. Nat. Genet. 10:288-293.

14. Liu, B., et al. 1998. Glutathione regulation of neutral sphingomyelinase in tumor necrosis factor-alpha-induced cell death. J. Biol. Chem. 273:11313-11320

15. Jayadev, S., Linardic, C.M., and Hannun, Y.A. 1994. Identification of arachidonic acid as a mediator of sphingomyelin hydrolysis in response to tumor necrosis factor alpha. J. Biol. Chem. 269:5757-5763.

16. Chan, T.A., Morin, P.J., Vogelstein, B., and Kinzler, K.W. 1998. Mechanisms underlying nonsteroidal antiinflammatory drug-mediated apoptosis. Proc. Natl. Acad. Sci. USA. 95:681-686.

17. Bose, R., et al. 1995. Ceramide synthase mediates daunorubicin-induced apoptosis: an alternative mechanism for generating death signals. Cell. 82:405-414.

18. Liao, W.C., et al. 1999. Ataxia telangiectasia-mutated gene product inhibits DNA damage-induced apoptosis via ceramide synthase. J. Biol. Chem. 274:17908-17917.

19. Li, C.M., et al. 2002. Insertional mutagenesis of the mouse acid ceramidase gene leads to early embryonic lethality in homozygotes and progressive lipid storage disease in heterozygotes. Genomics. 79:218-224.

20. Kohama, T., et al. 1998. Molecular cloning and functional characterization of murine sphingosine kinase. J. Biol. Chem. 273:23722-23728.

21. Liu, H., et al. 2000. Molecular cloning and functional characterization of a novel mammalian sphingosine kinase type 2 isoform. J. Biol. Chem. 275:19513-19520.

22. Cuvillier, O., et al. 1996. Suppression of ceramide-mediated programmed cell death by sphingosine-1-phosphate. Nature. 381:800-803.

23. Olivera, A., and Spiegel, S. 1993. Sphingosine-1-phosphate as 2 nd messenger in cell proliferation induced by PDGF and FCS mitogens. Nature. 365:557-560.

24. Hla, T., Lee, M.J., Ancellin, N., Paik, J.H., and Kluk, M.J. 2001. Lysophospholipids: receptor revelations. Science. 294:1875-1878.

25. El Bawab, S., et al. 2000. Molecular cloning and characterization of a human mitochondrial ceramidase. J. Biol. Chem. 275:21508-21513.

26. Kolesnick, R.N., Goni, F.M., and Alonso, A. 2000. Compartmentalization of ceramide signaling: physical foundations and biological effects. J. Cell. Physiol. 184:285-300.

27. Simons, K., and Ikonen, E. 2000. How cells handle cholesterol. Science. 290:1721-1726.

28. Prinetti, A., et al. 2001. Changes in the lipid turnover, composition, and organization, as sphingolipid-enriched membrane domains, in rat cerebellar granule cells developing in vitro. J. Biol. Chem. 276:21136-21145.

29. Yasuhara, S., et al. 1999. The 1999 Moyer award. Burn injury induces skeletal muscle apoptosis and the activation of caspase pathways in rats. J. Burn Care Rehabil. 20:462-470.

30. Bilderback, T.R., Grigsby, R.J., and Dobrowsky, R.T. 1997. Association of p75(NTR) with caveolin and localization of neurotrophin-induced sphingomyelin hydrolysis to caveolae. J. Biol. Chem. 272:10922-10927.

31.Zundel, W., and Giaccia, A. 1998. Inhibition of the anti-apoptotic PI(3)K/Akt/Bad pathway by stress. Genes Dev. 12:1941-1946.

32. Siegel, R.M., et al. 2000. Fas preassociation required for apoptosis signaling and dominant inhibition by pathogenic mutations. Science. 288:2354-2357.

33. Algeciras-Schimnich, A., et al. 2002. Molecular ordering of the initial signaling events of CD95. Mol. Cell. Biol. 22:207-220.

34. Gajate, C., and Mollinedo, F. 2001. The antitumor ether lipid ET-18$\mathrm{OCH}(3)$ induces apoptosis through translocation and capping of
Fas/CD95 into membrane rafts in human leukemic cells. Blood. 98:3860-3863.

35. Hueber, A.O., Bernard, A.M., Herincs, Z., Couzinet, A., and He, H.T. 2002. An essential role for membrane rafts in the initiation of Fas/CD95-triggered cell death in mouse thymocytes. EMBO Rep. 3:190-196.

36. Chalfant, C.E., et al. 2002. De novo ceramide regulates the alternative splicing of caspase 9 and Bcl-x in A549 lung adenocarcinoma cells. Dependence on protein phosphatase-1. J. Biol. Chem. 277:12587-12595.

37. Zhang, Y., et al. 1997. Kinase suppressor of Ras is ceramide-activated protein kinase. Cell. 89:63-72.

38. Yan, F., and Polk, D.B. 2001. Kinase suppressor of ras is necessary for tumor necrosis factor alpha activation of extracellular signal-regulated kinase/mitogen-activated protein kinase in intestinal epithelial cells. Cancer Res. 61:963-969.

39. Huwiler, A., Johansen, B., Skarstad, A., and Pfeilschifter, J. 2001. Ceramide binds to the CaLB domain of cytosolic phospholipase A2 and facilitates its membrane docking and arachidonic acid release. FASEB J. 15:7-9.

40. Heinrich, M., et al. 1999. Cathepsin D targeted by acid sphingomyelinasederived ceramide. EMBO J. 18:5252-5263.

41. Muller, G., et al. 1995. PKC zeta is a molecular switch in signal transduction of TNF-alpha, bifunctionally regulated by ceramide and arachidonic acid. EMBO J. 14:1961-1969.

42. Zhou, M., Horita, D.A., Waugh, D.S., Byrd, R.A., and Morrison, D.K. 2002. Solution structure and functional analysis of the cysteine-rich $\mathrm{C} 1$ domain of kinase suppressor of Ras (KSR). J. Mol. Biol. 315:435-446.

43. Huwiler, A., et al. 1996. Ceramide-binding and activation defines protein kinase c-Raf as a ceramide-activated protein kinase. Proc. Natl. Acad. Sci. USA. 93:6959-6963.

44. Salinas, M., Lopez-Valdaliso, R., Martin, D., Alvarez, A., and Cuadrado, A. 2000. Inhibition of PKB/Akt1 by C2-ceramide involves activation of ceramide- activated protein phosphatase in PC12 cells. Mol. Cell. Neurosci. 15:156-169.

45. Ruvolo, P.P., Deng, X., Ito, T., Carr, B.K., and May, W.S. 1999. Ceramide induces $\mathrm{Bcl} 2$ dephosphorylation via a mechanism involving mitochondrial PP2A. J. Biol. Chem. 274:20296-20300.

46. Morrison, D.K. 2001. KSR: a MAPK scaffold of the Ras pathway? J. Cell Sci. 114:1609-1612.

47. Deiss, L.P., Galinka, H., Berissi, H., Cohen, O., and Kimchi, A. 1996 Cathepsin D protease mediates programmed cell death induced by interferon-gamma, Fas/APO-1 and TNF-alpha. EMBOJ. 15:3861-3870.

48. Kashiwagi, K., Shirai, Y., Kuriyama, M., Sakai, N., and Saito, N. 2002 Importance of $\mathrm{C} 1 \mathrm{~B}$ domain for lipid messenger-induced targeting of protein kinase C. J. Biol. Chem. 277:18037-18045.

49. Charles, R., et al. 2000. Ceramide-coated balloon catheters limit neointimal hyperplasia after stretch injury in carotid arteries. Circ. Res. 87:282-288

50. Bourbon, N.A., Yun, J., Berkey, D., Wang, Y., and Kester, M. 2001. Inhibitory actions of ceramide upon PKC-epsilon/ERK interactions. Am. J. Physiol. Cell Physiol. 280:C1403-C1411.

51. Mehta, S., et al. 2000. Combined cytotoxic action of paclitaxel and ceramide against the human Tu138 head and neck squamous carcinoma cell line. Cancer Chemother. Pharmacol. 46:85-92.

52. Selzner, M., et al. 2001. Induction of apoptotic cell death and prevention of tumor growth by ceramide analogues in metastatic human colon cancer. Cancer Res. 61:1233-1240.

53. Potten, C.S. 1990. A comprehensive study of the radiobiological response of the murine (BDF1) small intestine. Int. J. Radiat. Biol. 58:925-973.

54. Paris, F., et al. 2001. Endothelial apoptosis as the primary lesion initiating intestinal radiation damage in mice. Science. 293:293-297.

55. Morita, Y., et al. 2000. Oocyte apoptosis is suppressed by disruption of the acid sphingomyelinase gene or by sphingosine-1-phosphate therapy. Nat. Med. 6:1109-1114.

56. Maurer, B.J., Melton, L., Billups, C., Cabot, M.C., and Reynolds, C.P. 2000 Synergistic cytotoxicity in solid tumor cell lines between N-(4-hydroxyphenyl)retinamide and modulators of ceramide metabolism. J. Natl. Can cer Inst. 92:1897-1909.

57. Wang, H., Maurer, B.J., Reynolds, C.P., and Cabot, M.C. 2001. N-(4hydroxyphenyl)retinamide elevates ceramide in neuroblastoma cell lines by coordinate activation of serine palmitoyltransferase and ceramide synthase. Cancer Res. 61:5102-5105.

58. Schwartz, G.K., et al. 1997. A pilot clinical/pharmacological study of the protein kinase $\mathrm{C}$-specific inhibitor safingol alone and in combination with doxorubicin. Clin. Cancer Res. 3:537-543.

59. Mandala, S., et al. 2002. Alteration of lymphocyte trafficking by sphingosine-1-phosphate receptor agonists. Science. 296:346-349.

60. Brinkmann, V., et al. 2001. FTY720 alters lymphocyte homing and protects allografts without inducing general immunosuppression. Transplant. Proc. 33:530-531.

61. Grassme, H., Jendrossek, V., Bock, J., Riehle, A., and Gulbins, E. 2002 Ceramide-rich membrane rafts mediate CD40 clustering. J. Immunol. 168:298-307. 